\title{
ON RESIDUALLY TRANSCENDENTAL VALUED FUNCTION FIELDS OF CONICS
}

\author{
by SUDESH K. KHANDUJA $\dagger$
}

(Received 26 November, 1992; revised 23 August 1994)

Dedicated to Professor I. S. Luthar on his 60th birthday.

1. Introduction. Let $K / K_{0}$ be a finitely generated field extension of transcendence degree 1 . Let $v_{0}$ be a valuation of $K_{0}$ and $v$ a valuation of $K$ extending $v_{0}$ such that the residue field of $v$ is a transcendental extension of the residue field $k_{0}$ of $v_{0}$; such a prolongation $v$ will be called a residually transcendental prolongation of $v_{0}$. By an element with the uniqueness property for $(K, v) /\left(K_{0}, v_{0}\right)$ (or more briefly for $\left.v / v_{0}\right)$ we mean an element $t$ of $K$ having $v$-valuation 0 which satisfies (i) the image of $t$ under the canonical homomorphism from the valuation ring of $v$ onto the residue field of $v$ (henceforth referred to as the $v$-residue ot $t$ ) is transcendental over $k_{0}$; that is $v$ coincides with the Gaussian valuation $v_{0}^{t}$ on the subfield $K_{0}(t)$ defined by $v_{0}^{t}\left(\sum_{i} a_{i} t^{i}\right)=\min _{i}\left(v_{0}\left(a_{i}\right)\right)$; (ii) $v$ is the only valuation of $K$ (up to equivalence) extending the valuation $v_{0}^{t}$.

In this paper, we consider the problem of existence and construction of elements with the uniqueness property for $(K, v) /\left(K_{0}, v_{0}\right)$ when $K$ is a function field of a conic over $K_{0}$; that is $K=K_{0}(x, y)$, where $(x, y)$ satisfies an irreducible polynomial relation of degree 2 over $K_{0}$ and $v$ is a residually transcendental extension of $v_{0}$.

In 1988, Polzin showed that the existence of elements with the uniqueness property for a residually transcendental extension $(K, v) /\left(K_{0}, v_{0}\right)$, where $K$ is a one variable function field over $K_{0}$ (any genus) for which the algebraic closure of $K_{0}$ in $K$ is a purely inseparable extension of $K_{0}$ and where the rank of $v$ is 1 , is equivalent to the so called "Local Skolem Property" (cf. [12]). His proof is based on rigid analytic geometry and uses a result of Matignon [7, p. 197, Thm. 3] which itself depends upon a structure theorem for affinoid domains proved in $[4$, p. 160 , Thm. 1]. For any genus $\geq 1$, he shows $[12$, p. 129 , Rk. 1] the existence of examples where the "Local Skolem Property" is not satisfied (this gives rise to valued function fields without the uniqueness property) and for genus 0 (i.e. function fields of conics [1, Chap. 16, Thm. 6]) he shows that the "Local Skolem Property" is always satisfied and so they have the uniqueness property. Our goal here has been to give an elementary proof of the result which not only shows the existence of an element with the uniqueness property but also gives a method to construct it explicitly. It may be remarked that in the particular case when $K$ is a simple transcendental extension of $K_{0}$ and rank $v$ is 1 , a direct proof based on valuation theory for the existence of elements with the uniqueness property has already been given by Matignon and $\mathrm{Ohm}$ (see [8, Thm. 0.1]).

We shall prove the following result.

THEOREM 1.1. Let $v_{0}$ be a rank 1 valuation of a field $K_{0}$. Let $K$ be a function field of $a$ conic over $K_{0}$ such that the algebraic closure of $K_{0}$ in $K$ is a purely inseparable extension

† The research is partially supported by CSIR vide grant no. 25/53/90-EMR-Il.

Glasgow Math. J. 38 (1996) 137-145. 
of $K_{0}$. Suppose that $v$ is a residually transcendental extension of $v_{0}$ to $K$. Then there exists an explicitly constructible element of $K$ which satisfies the uniqueness property for $v / v_{0}$.

The example in $\left[9\right.$, Cor. 2.3.4] shows that we cannot remove the condition " $v_{0}$ is of rank 1" from the hypothesis of Theorem 1.1.

2. Definitions, notations and some preliminary results. A field $K$, which is a function field of a conic over $K_{0}$, is said to be regular if (i) $K / K_{0}$ is separably generated and (ii) $K_{0}$ is algebraically closed in $K$.

Observe that a simple transcendental extension $K_{0}(t)$ of a field $K_{0}$ is a regular function field of a conic over $K_{0}$ which can be visualized by writing $K_{0}(t)$ as $K_{0}\left(t, t^{-1}\right)$, where $\left(t, t^{-1}\right)$ satisfies the equation $X Y-1=0$.

We shall abbreviate transcendental as tr., use $\mathbb{Z}$ for the set of integers and $X, Y$ for indeterminates.

As in [11], for any $a$ in the base field $K_{0}, H_{a}(X, Y)$ will stand for the polynomial $X^{2}-X Y-a Y^{2}$ or $X^{2}-a Y^{2}$ accordingly, depending on whether the characteristic of $K_{0}$ is 2 or not. It is a routine calculation to check that if $a$ and $b$ are non-zero elements of $K_{0}$, then the polynomial $H_{a}(X, Y)-b$ is irreducible over $K_{0}$.

The following Proposition 2.1 and Lemma 2.2 have been proved by an elementary method by Ohm (see [11, Thm. 2.3, Cor. 2.10]). We omit their proofs.

Proposition 2.1. Let $K$ be a regular function field of a conic over $K_{0}$. Then one can determine (by an explicit algorithm) non-zero elements $a$ and $b$ in $K_{0}$ such that $K=K_{0}(x, y)$, where $(x, y)$ satisfies $H_{a}(X, Y)-b=0$.

LEMMA 2.2. Let $K=K_{0}(x, y)$ be a regular function field of a conic over $K_{0}$ with $x, y$ related by $H_{a}(x, y)-b=0$, for some non-zero $a, b$ in $K_{0}$. Let $c$ be a root of the quadratic polynomial $H_{a}(X, 1)$. Then $K(c)$ is a simple transcendental extension of $K_{0}(c)$ having $z=x-$ cy as a generator.

We now prove the following result.

LEMMA 2.3. Let $K_{0}, K, c$ and $z$ be as in the above lemma. Let $v_{0}$ be a rank 1 valuation of $K_{0}$ with residue field $k_{0}$ and $w$ a residually transcendental prolongation of $v_{0}$ to $K(c)$. Let $s$ be the smallest positive integer such that $w\left(z^{s}\right)=w(q)$, for some $q$ in $K_{0}(c)$. If the $w$-residue of $z^{s} / q$ is transcendental over $k_{0}$, then one can explicitly construct an element of $K$ which satisfies the uniqueness property for $v / v_{0}$, where $v$ is the valuation of $K$ obtained by restricting $w$.

Proof. We may assume that $c \notin K_{0}$, for if $c \in K_{0}$ then as in [10, Prop. 4.3] it can be easily seen that $z^{s} / q$ satisfies the uniqueness property for $v / v_{0}$. Let $\bar{c}$ denote the other root of $H_{a}(X, 1), \bar{z}$ the element $x-\bar{c} y$ and $\sigma$, the unique non-trivial automorphism of $K(c) / K$ defined by $\sigma(c)=\bar{c}$. We denote $z^{s} / q$ by $\xi$, the $w$-residue of any element $\eta$ in the valuation ring of $w$ by $\eta^{*}$ and the residue field of $w$ by $l$. Two cases are distinguished.

Case $I$. $v_{0}$ has unique extension, say $w_{0}$ to $K_{0}(c)$. 
We shall prove the following statements.

(i) If $w(\sigma(\xi)) \geq 0$, then $\xi+\sigma(\xi)$ satisfies the uniqueness property for $v / v_{0}$.

(ii) If $w(\sigma(\xi))<0$, then $\xi^{-1}+\sigma\left(\xi^{-1}\right)$ has the above property.

Proof of (i). Set $t=\xi+\sigma(\xi)$. First consider the possibility when $w(\sigma(\xi))=0$. Using the equality $z \bar{z}=b$, it is easy to see that in the present situation $v_{0}\left(b^{s} / q \sigma(q)\right)=0$ and $t^{*}=\xi^{*}+\left(b^{s} / q \sigma(q)\right)^{*} \xi^{*-1}$. Hence, if $l_{0}$ is the residue field of $w_{0}$, then

$$
\left[l: l_{0}\left(t^{*}\right)\right] \geq\left[l_{0}\left(\xi^{*}\right): l_{0}\left(t^{*}\right) !=2 .\right.
$$

Applying Luroth's Lemma [13, p. 197], we see that

$$
\left[K(c): K_{0}(c, t)\right]=\left[K_{0}(c, z): K_{0}(c, t)\right]=2 s \text {. }
$$

As in [10, Prop. 4.3], it can be easily seen that the value group of $w$ is $H_{0}+\mathbb{Z}\left(w_{0}(q) / s\right)$, where $H_{0}$ is the value group of $w_{0}$. Thus if $w_{0}^{t}$ denotes the Gaussian valuation obtained by restricting $w$ to $K_{0}(c, t)$, then the index of ramification of $w / w_{0}^{t}$ is $s$. It now follows from (1), (2) and the fundamental inequality [2, §8.3, Thm. 1] that $w$ is the only prolongation of $w_{0}^{t}$ to $K(c)$. By virtue of the hypothesis of Case I, the Gaussian valuation $v_{0}^{t}$ of $K_{0}(t)$ can be uniquely extended to $K_{0}(c, t)$. This $w$ is the only prolongation of $v_{0}^{t}$ to $K(c)$ which implies that $v$ is the unique prolongation of $v_{0}^{t}$ to $K$ as desired.

Consider now the situation when $w\left(\sigma(\xi)>0\right.$; then $t^{*}=\xi^{*}$. The valuations $w$ and $w \circ \sigma$ of $K(c)$ are different and both extend $v_{0}^{t}$. Since $w_{0}^{t}$ is the only extension of $v_{0}^{t}$ to $K_{0}(c, t)$, we conclude that $w \circ \sigma$ also extends $w_{0 .}^{t}$. Clearly the index of ramification of $w \circ \sigma / w_{0}^{t}$ is the same as that of $w / w_{0}^{t}$ which (as mentioned above in the proof of (i)) is $s$. It now follows from the fundamental inequality [2, $\$ 8.3, T h m .1]$ and (2) that $w$ and $w \circ \sigma$ are the only extensions of $w_{0}^{t}$ to $K(c)$. Since these valuations coincide with $v$ on $K$, it is the unique extension of $v_{0}^{t}$ to $K$.

Proof of (ii). Proceeding exactly as in the above paragraph, one can prove (ii). We omit the proof.

Case II. There are two extensions of $v_{0}$ to $K_{0}(c)$.

Let $w_{0}$ denote the prolongation of $v_{0}$ to $K_{0}(c)$ obtained by restricting $w$, and $\vec{w}_{0}$ be the other prolongation; then $\bar{w}_{0}(d)=w_{0}(\sigma(d))$ for all $d \in K_{0}(c)$. By the Independence Theorem [2, Cor. 3.14], we can choose $d \in K_{0}(c)$ such that

$$
w_{0}(d)=0, \quad \bar{w}_{0}(d)=v_{0}(q \sigma(q))-v_{0}\left(b^{s}\right) .
$$

Set $t=d \xi+\sigma(d \xi)$. Keeping in view (3) one can easily verify that

$$
t^{*}=d^{*} \xi^{*}+\left(\sigma(d) b^{s} / q \sigma(q)\right)^{*} \xi^{*-1},
$$

and hence $\left[l: k_{0}\left(t^{*}\right)\right] \geq 2$. Arguing exactly as in the proof of assertion (i) of Case $\mathrm{I}$, it can be shown that $w$ is the only prolongation of $w_{0}^{i}$ to $K(c)$. Since the restriction of the valuation $w \circ \sigma$ to $K_{0}(c, t)$ is the Gaussian valuation $\bar{w}_{0}^{t}$, we conclude that $w \circ \sigma$ is the unique prolongation of $\bar{w}_{0}^{t}$ to $K(c)$. Thus $w$ and $w \circ \sigma$ are the only prolongations of $v_{0}^{t}$ to $K(c)$. As these valuations agree with $v$ on $K$, it is the unique extension of $v_{0}^{t}$ to $K$.

Definition. For a finite extension $(F, u) /\left(F_{0}, u_{0}\right)$ of valued fields, the henselian defect is defined to be $\left[F^{h}: F_{0}^{h}\right] / e f$, where " $h$ " stands for the henselisation with respect to the underlying valuation and $e, f$ for the ramification index and the residual degree of $u / u_{0}$. 
A FUNDAMENTAL EQUALITY. If $u_{0}$ is a valuation of a field $F_{0}$ and $u_{1}, \ldots, u_{m}$ are all the non-equivalent prolongations of $u_{0}$ to a finite extension $F$ of $F_{0}$, then it is well known (see $[2$, p. $125(17.3)])$ that

$$
\begin{aligned}
{\left[F: F_{0}\right] } & =\sum_{i=1}^{m}\left[\left(F, u_{i}\right)^{h}:\left(F_{0}, u_{0}\right)^{h}\right] \\
& =\sum_{i=1}^{m} e_{i} f_{i} d_{i},
\end{aligned}
$$

where " $h$ " stands for henselisation and $e_{i}, f_{i}, d_{i}$ denote respectively the ramification index, the residual degree and the henselian defect of the extension $u_{i} / u_{0}$.

Notations. Let $w_{0}$ be a valuation of a field $L_{0}$ with value group $H_{0}$ and residue field $l_{0}$. Let $w$ be a residually tr. prolongation of $w_{0}$ to a simple tr. extension $L=L_{0}(z)$ having value group $H$ and residue field $l$. As in [9], we shall denote by $E, I, R$ (more precisely by $E\left(w / w_{0}\right)$ etc. $)$ the numbers defined by

$$
\begin{aligned}
E & =\min \left\{\left[L: L_{0}(\xi)\right] \mid \xi \in L, w(\xi) \geq 0, \xi^{*} \operatorname{tr} \text {. over } l_{0}\right\}, \\
I & =\left[H: H_{0}\right], \\
R & =\left[\Delta: l_{0}\right], \text { where } \Delta \text { is the algebraic closure of } l_{0} \text { in } l .
\end{aligned}
$$

Let $t$ be an element of the valuation ring of $w$ such that $t^{*}$ is tr. over $l_{0}$. We shall denote by $D^{h}$, the henselian defect of the finite extension $(L, w) /\left(L_{0}(t), w_{0}^{t}\right):\left(w_{0}^{t}\right.$ denotes the restriction of $w$ to $L_{0}(t)$ ); in view of [9, Thm. 2.2], $D^{h}$ is independent of the choice of residually tr. element $t$.

The following result of Matignon and Ohm (whose proof is omitted) is quoted for future reference (see $[9$, Cor. 2.3.2]).

THEOREM 2.4. Let $L_{0} \subset L, w_{0}, w, E, I, R$, and $D^{h}$ be as above.

(i) There exists an element of $L$ which satisfies the uniqueness property for $w / w_{0}$ if and only if $E=I R D^{h}$ holds.

(ii) If $w_{0}$ is of rank 1 , then any residually transcendental element $t$ of $L$ for which $\left[L: L_{0}(t)\right]$ is $E$, satisfies the uniqueness property for $w / w_{0}$.

We now recall some results from [5]. Let $L_{0} \subset L=L_{0}(z), w_{0}, w, l_{0} \subseteq l, H_{0} \subseteq H$ and $E$ be as above. Fix an algebraic closure $L_{0}^{\prime}$ of $L_{0}$ and a prolongation $w^{\prime}$ of $w$ to $L_{0}^{\prime}(z)$. Denote the restriction of $w^{\prime}$ to $L_{0}^{\prime}$ by $w_{0}^{\prime}$ and the $w^{\prime}$-residue of an element $\eta$ in the valuation ring of $w^{\prime}$ by $\eta^{*}$. As in [10, p. 205, 2.5], it can be easily seen that there exist $\alpha$ and $a^{\prime}$ in $L_{0}^{\prime}$ such that $\left((z-\alpha) / a^{\prime}\right)^{*}$ is tr. over $l_{0}$. We denote $w^{\prime}(z-\alpha)$ by $\mu$. Therefore for any $f(z)=\sum c_{i}^{\prime}(z-\alpha)^{i}$ in $L_{0}^{\prime}[z]$, we have

$$
w^{\prime}(f(z))=\min _{i}\left(w_{0}^{\prime}\left(c_{i}^{\prime}\right)+i \mu\right)
$$

in particular if $\gamma \in L_{0}^{\prime}$, then

$$
w^{\prime}(z-\gamma)=\min \left(w_{0}^{\prime}(\alpha-\gamma), \mu\right) .
$$

Define a subset $D^{\prime}$ of $L_{0}^{\prime}$ by

$$
D^{\prime}=\left\{\gamma \in L_{0}^{\prime} \mid w_{0}^{\prime}(\gamma-\alpha) \geq \mu\right\}
$$


Choose an element $\beta \in D^{\prime}$ such that $\left[L_{0}(\beta): L_{0}\right] \leq\left[L_{0}(\gamma): L_{0}\right]$ for all $\gamma$ in $D^{\prime}$. Let $P(X)$ denote the minimal polynomial of $\beta$ over $L_{0}$ of degree $n$ (say); its roots $\beta=\beta_{1}, \ldots, \beta_{n}$ are arranged so that $w_{0}^{\prime}\left(\alpha-\beta_{i}\right) \geq \mu$ for $1 \leq i \leq m$ and $w_{0}^{\prime}\left(\alpha-\beta_{i}\right)<\mu$ for $i \geq m+1$. It follows from $\left(5^{\prime}\right)$ that

$$
w(P(z))=\sum_{i=1}^{n} w^{\prime}\left(z-\beta_{i}\right)=m \mu+\sum_{i=m+1}^{n} w_{0}^{\prime}\left(\alpha-\beta_{i}\right)=\theta \quad \text { (say). }
$$

Let $s$ be the smallest positive integer such that $s \theta \in H_{1}$, where $H_{1}$ is the value group of the valuation $w_{0}^{\prime}$ restricted to $L_{0}(\beta)$. We can choose a polynomial $q(z) \in L_{0}[z]$ of degree less than $n$ satisfying

$$
w\left(P(z)^{s}\right)=w_{0}^{\prime}(q(\beta)) \text {. }
$$

By assertion (ii) of [5, Lemma 2.1], for any polynomial $g(z)$ in $L_{0}^{\prime}[z]$, none of whose roots is in $D^{\prime}$, one has

$$
w^{\prime}(g(z)-g(\beta))>w^{\prime}(g(z)) \text {. }
$$

Since $q(z) \in L_{0}[z]$, being of degree less than $n$, has no root in $D^{\prime}$, it follows from (6) and (7) that $w\left(P(z)^{s}\right)=w(q(z))$. We shall denote $P(z)^{s} / q(z)$ by $\xi$. By virtue of (5) and $\left[5\right.$, Thms. 1.4, 1.3] the $w$-residue of $\xi$ is tr. over $l_{0}$ and the residue field $l$ of $w$ is given by

$$
l=\Delta\left(\xi^{*}\right),
$$

where $\Delta$ is the residue field of the valuation obtained by restricting $w_{0}^{\prime}$ to $L_{0}(\beta)$. It is also clear from the proof of $[5, T h m .1 .3(i)]$ that

$$
E=E\left(w / w_{0}\right)=s n
$$

With the above notations we prove two lemmas.

LeMma 2.5. If $\gamma$ is an element of $D^{\prime}$ which is algebraic of degree $n$ over $L_{0}$, then its minimal polymomial $F(X)$ over $L_{0}$ satisfies $w(P(z))=w(F(z))$ and $\left(P(z)^{s} / q(z)\right)^{*}=$ $\left(F(z)^{s} / q(z)\right)^{*}+\delta$, for some $\delta$ algebraic over $l_{0}$.

Proof. Suppose to the contrary that $w(P(z)) \neq w(F(z))$. On interchanging the roles of $\beta$ and $\gamma$ if necessary, assume that $w(P(z))<w(F(z))$. Then the $w$-residue of $\left(P(z)^{s}-F(z)^{s}\right) / q(z)$ equals that of $P(z)^{s} / q(z)$ and hence is tr. over $l_{0}$, which is impossible as both $P(z)^{s}-F(z)^{s}$ and $q(z)$ are of degree less than $E=s n$. For the same reason, the $w$-residue of $\left(P(z)^{s}-F(z)^{s}\right) / q(z)$ must be algebraic over $l_{0}$.

The following lemma can be quickly proved using (7) and the strong triangle law. We omit its proof.

LEMMA 2.6. If $\eta=g(z) / h(z) \in L_{0}^{\prime}(z)$ is such that $w^{\prime}(\eta)=0$ and no root of the polynomial $g(z) h(z)$ is in $D^{\prime}$, then $\eta^{*}=(g(\beta) / h(\beta))^{*}$.

3. Proof of Theorem 1.1. Since any valuation of a field has a unique prolongation to a purely inseparable extension, it may be assumed that $K_{0}$ is algebraically closed in $K$. Without loss of generality, we may further assume that $K$ is a regular function field of a 
conic over $K_{0}$, for if $K=K_{0}\left(x^{\prime}, y^{\prime}\right)$, where $x^{\prime}, y^{\prime}$ satisfy an irreducible polynomial relation of degreee 2 over $K_{0}$, then the assumption that $K / K_{0}$ is not separably generated would lead to $y^{\prime}$ being algebraic and purely inseparable over $K_{0}\left(x^{\prime}\right)$ and the theorem needs to be proved for $K_{0}\left(x^{\prime}\right) / K_{0}$. From now on, $K$ is as in Lemma 2.2; $c, \bar{c}, \sigma$ are as introduced in the opening lines of the proof of Lemma 2.3; and $\bar{\eta}$ will stand for $\sigma(\eta)$ for any $\eta$ in $K(c)$. In view of Theorem 2.4(ii), we need to prove the theorem only when $c \notin K_{0}$.

We fix a prolongation $w^{\prime}$ of $v$ to $K K_{0}^{\prime}=K_{0}^{\prime}(z)$, where $K_{0}^{\prime}$ is a fixed algebraic closure of $K_{0}$. Let $w_{0}, w_{0}^{\prime}, w$ be the valuations obtained by restricting $w^{\prime}$ to $K_{0}(c), K_{0}^{\prime}$, and $K(c)$ respectively. We set $L_{0}=K_{0}(c), L=L_{0}(z)=K(c)$ and retain the notations $H_{0}, H, l_{0}, l, E$, $I, R, D^{h}$ introduced in the lines preceding Theorem 2.4; by this theorem we have

$$
E=I R D^{h}
$$

Let $\alpha, \mu, D^{\prime}, \beta, P(X)=\sum_{i=0}^{n-1} c_{i} X^{i}+X^{n}, s, q(X)$ and $\xi=P(z)^{s} / q(z)$ be as in the paragraph preceding Lemma 2.5. Observe that if $0 \in D^{\prime}$ then $w_{0}^{\prime}(\alpha) \geq \mu=w_{0}^{\prime}\left(a^{\prime}\right)$, which imples that $\left(z / a^{\prime}\right)^{*}=\left((z-\alpha) / a^{\prime}\right)^{*}+\left(\alpha / a^{\prime}\right)^{*}$ is tr. over $l_{0}$ and the proof of the theorem will be complete by virtue of Lemma 2.3. From now on it is assumed that $0 \notin D^{\prime}$.

Since $z \bar{z}=b$, the monic polynomial $P_{1}(z)$, defined by

$$
\sigma(P(z))=\sum_{i=0}^{n-1} \bar{c}_{i} \bar{z}^{i}+\bar{z}^{n}=\bar{c}_{0} P_{1}(z) / z^{n},
$$

has degree $n$ and is not divisible by $z$. If $r$ denotes the degree of the polynomial $q(z)$, then it can be easily seen that $\sigma(q(z))=q_{1}(z) / z^{r}$, where $q_{1}(z)$ is a polynomial of degree at most $r$. Consequently

$$
\sigma(\xi)=P_{1}(z)^{s} / h(z),
$$

where $h(z)=\bar{c}_{0}^{-s} z^{s n-r} q_{1}(z)$ is a polynomial of degree at most $s n$ none of whose roots is in $D^{\prime}$. It now follows from Luroth's Lemma and (9) that

$$
\left[K_{0}(c, z): K_{0}\left(c, \xi+\xi^{-}\right)\right] \leq 2 s n=2 E,
$$

which in view of (10) yields

$$
\left[K_{0}(c, z): K_{0}\left(c, \xi+\xi^{-}\right)\right] \leq 2 I R D^{h} .
$$

Arguing similarly, we see that

$$
\left[K_{0}(c, z): K_{0}\left(c, \xi \xi^{-}\right)\right] \leq 2 I R D^{h} .
$$

Two cases are distinguished.

Case I. $w_{0}$ is the unique prolongation of $v_{0}$ to $K_{0}(c)$. In this case, we shall prove the following statements.

(i) If $w(\sigma(\xi)) \geq 0$ and $\sigma(\xi)^{*}$ is algebraic over $l_{0}$, then $\xi+\sigma(\xi)$ satisfies the uniqueness property for $v / v_{0}$.

(ii) If $w(\sigma(\xi))<0$, then $\xi^{-1}+\sigma\left(\xi^{-1}\right)$ satisfies the above property.

(iii) If $w(\sigma(\xi))=0$ and $\xi^{*}$ is tr. over $l_{0}$, then $\xi \sigma(\xi)$ satisfies this property.

Proof of $(i)$. Set $t=\xi+\sigma(\xi)$. By hypothesis $t^{*}-\xi^{*}$ is in the algebraic closure $\Delta$ of $l_{0}$ in $l$. Recall that $R=\left[\Delta: l_{0}\right]$ and, by $(8), l=\Delta\left(\xi^{*}\right)=\Delta\left(t^{*}\right)$. Let $v_{0}^{t}, w_{0}^{t}$ denote the Gaussian 
valuations of $K_{0}(t), K_{0}(c, t)$ respectively obtained by restricting $w$. It is immediate from the hypothesis of Case I that $w_{0}^{t}$ is the unique prolongation of $v_{0}^{t}$ to $K_{0}(c, t)$. Thus the valuation $w \circ \sigma$, being an extension of $\nu_{0}^{t}$, also extends $w_{0}^{t}$. Since $\sigma$ maps $K_{0}(c, t)$ onto itself, it follows that the index of ramification, the residual degree and henselian defect of the extension $w \circ \sigma / w_{0}^{t}$ are the same as the corresponding ones of $w / w_{0}^{t}$, which are $I, R$, $D^{h}$ respectively. In view of (4) and (12), we conclude that $w$ and $w \circ \sigma$ are the only prolongations of $w_{0}^{t}$ (and hence of $v_{0}^{t}$ ) to $K(c)=K_{0}(c, z)$. It is now clear that their restriction $v$ to $K$ is the unique extension of $v_{0}^{t}$, which proves (i).

The proof of (ii) is similar and is omitted.

Proof of (iii). Here we denote $\xi \sigma(\xi)$ by $t$. In view of $(11), \sigma(\xi)=P_{1}(z)^{s} / h(z)$, where $h(z)$ is a polynomial having no root in $D^{\prime}$. Since $\sigma(\xi)^{*}$ is given to be tr. over $l_{0}$, it follows from Lemma 2.6 that at least one of the roots of $P_{1}(X)$, say $\gamma$, is in $D^{\prime}$. As each element of $D^{\prime}$ is algebraic over $K_{0}(c)$ of degree not less than $n$, we see that $P_{1}(X)$ is the minimal polynomial of $\gamma$ over $K_{0}(c)$. It is now immediate from Lemmas 2.5, 2.6 that

$$
\sigma(\xi)^{*}=\left(\left(P_{1}(z)\right)^{s} / q(z)\right)^{*}(q(\beta) / h(\beta))^{*}=\left(\xi^{*}+\delta\right) \delta^{\prime},
$$

for some $\delta, \delta^{\prime}$ in $\Delta$. Hence the residual degree of $w / w_{0}^{t}$ is $\left[\Delta\left(\xi^{*}\right): l_{0}\left(t^{*}\right)\right]=2 R$. Since the index of ramification of $w / w_{0}^{t}$ is $I$ and its henselian defect is $D^{h}$, we conclude by virtue of (4) and (13) that $w$ is the only extension of $w_{0}^{t}$ (and hence of $v_{0}^{t}$ ) to $K(c)$, which immediately gives (iii).

Case II. $v_{0}$ has two extensions to $K_{0}(c)$. Let $w_{0}, \bar{w}_{0}$ be two extensions of $v_{0}$ to $K_{0}(c)$; then $\bar{w}_{0}(d)=w_{0}(\sigma(d))$, for all $d$ in $K_{0}(c)$. We split into two sub cases.

Sub case $(i) . P_{1}(X)$ has a root, say $\gamma$ in $D^{\prime}$. Here $P_{1}(X)$ is the minimal polynomial of $\gamma$ over $K_{0}(c)$. Let $j$ be a positive integer such that $j w(\sigma(\xi))$ is in the value group of $v_{0}$. By the Independence Theorem [3, Cor. 3.14], there exists $d \in K_{0}(c)$ satisfying

$$
w_{0}(d)=0, \quad w_{0}^{-}(d)=-j w(\sigma(\xi)) .
$$

We show that $t=d \xi^{j} \sigma\left(d \xi^{j}\right)$ satisfies the uniqueness property for $v / v_{0}$. In view of $(14), w(t)=0$. Using (11) and Lemmas 2.5, 2.6, it can be easily seen that

$$
t^{*}=\xi^{* j}\left(\xi^{*}+\delta\right)^{j} \delta^{\prime},
$$

for some $\delta, \delta^{\prime}$ in $\Delta$; consequently the residual degree of $w / w_{0}^{t}$ is given by

$$
\left[\Delta\left(\xi^{*}\right): l_{0}\left(t^{*}\right)\right]=\left[\Delta\left(\xi^{*}\right): \Delta\left(t^{*}\right)\right]\left[\Delta\left(t^{*}\right): l_{0}\left(t^{*}\right)\right]=2 j R .
$$

Applying Luroth's Lemma, (9) and (10), we see that

$$
\left[K_{0}(c, z): K_{0}(c, t)\right] \leq 2 j s n=2 j I R D^{h} .
$$

Arguing exactly as in the proof of assertion (iii) of Case I, and using (15) and (16), we conclude that $w$ is the unique extension of $w_{0}^{t}$ to $K(c)$. Since the restriction of $w \circ \sigma$ to $K_{0}(c, t)$ is $\bar{w}_{0}^{t}$, it follows that $w \circ \sigma$ is the unique extension of $\bar{w}_{0}^{i}$ to $K(c)$. Thus, $w, w \circ \sigma$ are the only valuations of $K(c)$ (and hence $v$ is the only valuation of $K$ ) extending $v_{0}^{t}$.

Sub case (ii). No root of $P_{1}(X)$ is in $D^{\prime}$. In view of sub case (i) of Case II, it is enough to construct an element $\gamma \in K_{0}(c, \beta) \cap D^{\prime}$ whose minimal polynomial $Q(X)$ 
(say) over $K_{0}(c)$ is such that at least one root of the monic polynomial $Q_{1}(X)$ of degree $n$ is in $D^{\prime}$, where $Q_{1}(z)$ is defined by

$$
\sigma(Q(z))=d Q_{1}(z) / z^{n}, \quad d \in K_{0}(c) .
$$

To construct $\gamma$, we consider two possibilities. Suppose first that $c \notin K_{0}(\beta)$. Let $\tau$ denote the automorphism of $K_{0}(c, \beta) / K_{0}(\beta)$ defined by $\tau(c)=\bar{c}$ and $w_{1}$ the valuation of $K_{0}(c, \beta)$ obtained by restricting $w^{\prime}$. By the Approximation Theorem [3, Thm. 3.13], applied to the valuations $w_{1}, w_{1} \circ \tau$, there exists $\gamma_{1} \in K_{0}(c, \beta)$ such that

$$
\begin{gathered}
w_{1}\left(\gamma_{1}-\beta^{-1}\right)>\max \left(w_{1}\left(\beta^{-1}\right), \mu-2 w_{1}(\beta)\right), \\
w_{1} \circ \tau\left(\gamma_{1}-\beta b^{-1}\right)>\mu-v_{0}(b) .
\end{gathered}
$$

Set $\gamma=1 / \gamma_{1}$. It is immediate from (18) that $w_{1}(\beta)=w_{1}(\gamma)$ and $w_{1}(\beta-\gamma)>\mu$. We show that this choice of the element $\gamma$ of $K_{0}(c, \beta) \cap D^{\prime}$ works. In view of (19), we have

$$
w_{1}\left(b \tau\left(\gamma^{-1}\right)-\beta\right)>\mu,
$$

which implies that $b \tau\left(\gamma^{-1}\right) \in D^{\prime}$. Hence the proof of the desired assertion in the present situation is complete once we show that $b \tau\left(\gamma^{-1}\right)$ is a root of $Q_{1}(z)$, where $Q_{1}(z)$ is as in (17). Let $\sigma^{\prime}$ be an automorphism of $K K_{0}^{\prime} / K$ which coincides with $\tau$ on $K_{0}(c, \beta)$; such an automorphism exists, because $K$ being a regular extension of $K_{0}$ is linearly disjoint from $K_{0}^{\prime}$ over $K_{0}$; (see [6, Chp. 3, §1, Thm. 2]). On writing $Q(z)$ as $\prod_{i=1}^{n}\left(z-\delta_{i}\right)$ with $\delta_{1}=\gamma$ we see that

$$
\sigma(Q(z))=\prod_{i=1}^{n}\left(\bar{z}-\sigma^{\prime}\left(\delta_{i}\right)\right)=\prod_{i=1}^{n}\left(b-z \sigma^{\prime}\left(\delta_{i}\right)\right) / z^{n},
$$

which shows that $b \sigma^{\prime}\left(\delta_{1}^{-1}\right)=b \tau\left(\gamma^{-1}\right)$ is a root of $Q_{1}(z)$.

The remaining case "when $c \in K_{0}(\beta)$ " will be disposed of by showing that we can find $\beta^{\prime} \in D^{\prime}$ which is algebraic of degree $n$ over $K_{0}(c)$ such that $c \notin K_{0}\left(\beta^{\prime}\right)$.

Since the valuation $v_{0}$ has two extensions to the separable quadratic extension $K_{0}(c)$ of $K_{0}$, it follows from [3, Thm. 2.12] that $c \in \widehat{K}_{0}$, where $\widehat{K}_{0}$ is the completion of $K_{0}$ with respect to $v_{0}$. As $K_{0}$ is dense in $\hat{K}_{0}$, corresponding to the coefficients $c_{0}, c_{1}, \ldots$ of $P(X)$ we can choose $a_{0}, a_{1}, \ldots$ in $K_{0}$ such that

$$
w_{0}\left(c_{i}-a_{i}\right)>n \mu+i \lambda \quad(0 \leq i \leq n-1),
$$

where $\lambda=\max \left(w^{\prime}(\beta),-w^{\prime}(\beta)\right)$. Set

$$
R(X)=\sum_{i=0}^{n-1} a_{i} X^{i}+X^{n}
$$

We claim that there exists a root $\beta^{\prime}$ of $R(X)$ such that $w^{\prime}\left(\beta-\beta^{\prime}\right)>\mu$. Suppose not; then for each root $\alpha_{i}$ of $R(X), w^{\prime}\left(\beta-\alpha_{i}\right) \leq \mu$; consequently

$$
w^{\prime}(P(\beta)-R(\beta))=w^{\prime}(R(\beta))=\sum_{i=1}^{n} w^{\prime}\left(\beta-\alpha_{i}\right) \leq n \mu ;
$$


this is impossible because in view of (20)

$$
w^{\prime}(P(\beta)-R(\beta))=w^{\prime}\left(\sum_{i=0}^{n-1}\left(c_{i}-a_{i}\right) \beta^{i}\right) \geq \min _{i}\left(w^{\prime}\left(c_{i}-a_{i}\right)-i \lambda\right)>n \mu .
$$

Hence the claim.

It only remains to be shown that $c \notin K_{0}\left(\beta^{\prime}\right)$; for otherwise we have

$$
2 n \leq\left[K_{0}\left(c, \beta^{\prime}\right): K_{0}(c)\right]\left[K_{0}(c): K_{0}\right]=\left[K_{0}\left(\beta^{\prime}\right): K_{0}\right] \leq n .
$$

The proof of the theorem is now complete.

\section{REFERENCES}

1. E. Artin, Algebraic numbers and algebraic functions (Gordon and Breach, New York 1967). 1972).

2. N. Bourbaki, Commutative algebra, Chapter 6 (Hermann Publishers in Arts and Science,

3. O. Endler, Valuation theory (Springer-Verlag, 1972).

4. J. Fresnel and M. Matignon, Sur les espaces analytiques quasicompacts de dimension 1 sur un corps value complet, ultrametrique, Annali di Mathematica pura ed applicata (4), 145 (1986), 159-210.

5. S. K. Khanduja, On valuations of $K(x)$, Proc. Edinburgh Math. Soc. 35 (1992), 419-426.

6. S. Lang, Introduction to algebraic geometry (Interscience publishers, 1964).

7. M. Matignon, Genre et genre residual des corps de fonctions values, Manuscripta Math. 58 (1987), 179-214.

8. M. Matignon and J. Ohm, A structure theorem for simple transcendental extensions of valued fields, Proc. Amer. Math. Soc. 104 (1988), 392-402.

9. M. Matignon and J. Ohm, Simple transcendental extensions of valued fields III: The uniqueness property, J. Math. Kyoto Univ., 30 (1990), 347-365. 201-221.

10. J. Ohm, Simple transcendental extensions of valued fields, J. Math. Kyoto Univ., 22 (1982),

11. J. Ohm, Function fields of conics, a theorem Amitsur-MacRae and a problem of Zariski, in Algebraic geometry and its applications, (Springer-Verlag, 1994), 333-363.

12. M. Polzin, Prolongement de la valeur absolue de Gauss et probleme de Skolem, Bull. Soc. Math. France 116 (1988), 103-132.

13. B. L. van der Waerden, Modern algebra 1 (Ungar, New York, 1964).

Centre for Advanced Study in Mathematics

PUNJAB University

Chandigarh 160014

INDIA 